\title{
An Abstraction of Whitney's Broken Circuit Theorem
}

\author{
Klaus Dohmen \\ Department of Mathematics \\ Mittweida University of Applied Sciences \\ Mittweida, Germany \\ dohmen@hs-mittweida.de
}

\author{
Martin Trinks \\ Center for Combinatorics \\ Nankai University \\ Tianjin, China \\ martin.trinks@googlemail.com
}

Submitted: May 8, 2014; Accepted: Nov 3, 2014; Published: Nov 13, 2014

Mathematics Subject Classifications: 05A15, 05C30, 05C31, 06A07, 11A25, 52A01

\begin{abstract}
We establish a broad generalization of Whitney's broken circuit theorem on the chromatic polynomial of a graph to sums of the type $\sum_{A \subseteq S} f(A)$ where $S$ is a finite set and $f$ is a mapping from the power set of $S$ to an abelian group. We give applications to the domination polynomial and the subgraph component polynomial of a graph, the chromatic polynomial of a hypergraph, the characteristic polynomial and Crapo's beta invariant of a matroid, and the principle of inclusion-exclusion. Thus, we discover several known and new results in a concise and unified way. As further applications of our main result, we derive a new generalization of the maximums-minimums identity and of a theorem due to Blass and Sagan on the Möbius function of a finite lattice, which generalizes Rota's crosscut theorem. For the classical Möbius function, both Euler's totient function and its Dirichlet inverse, and the reciprocal of the Riemann zeta function we obtain new expansions involving the greatest common divisor resp. least common multiple. We finally establish an even broader generalization of Whitney's broken circuit theorem in the context of convex geometries (antimatroids).
\end{abstract}

Keywords: graph polynomial; domination polynomial; beta invariant; broken circuit; Möbius function; lattice; max-min identity; totient; Dirichlet inverse; Riemann zeta function; closure system; convex geometry

\section{Introduction}

Whitney's broken circuit theorem [29] is one of the most significant results on the chromatic polynomial of a graph. We refer to Diestel [9] for general graph terminology, and 
to Dong, Koh and Teo [14] for a comprehensive treatment of the chromatic polynomial. The chromatic polynomial of any finite simple graph $G=(V, E)$ can be expressed as

$$
P(G, x)=\sum_{A \subseteq E}(-1)^{|A|} x^{c(V, A)}
$$

where $c(V, A)$ denotes the number of connected components of the spanning subgraph $(V, A)$. The significance of the chromatic polynomial lies in the fact that for any $x \in \mathbb{N}$ it evaluates to the number of proper $x$-colourings of $G$, that is, the number of mappings $f: V \rightarrow\{1, \ldots, x\}$ such that $f(v) \neq f(w)$ for any edge $\{v, w\} \in E$. This interpretation matches the original definition due to Birkhoff [5], whereas the expansion in Eq. (1) goes back to Whitney [29]. In this paper, we adopt Eq. (1) as a definition.

In order to state Whitney's broken circuit theorem, we assume that the edge set of $G$ is endowed with a linear ordering relation. Given a set $C$ consisting of the edges of a cycle of $G$, we refer to $C \backslash\{\max C\}$ as a broken circuit of $G$. Thus, a broken circuit of $G$ is obtained from the edge set of a cycle of $G$ by removing its maximum edge.

In his prominent result, Whitney [29] showed that the sum in Eq. (1) can be restricted to those subsets $A$ which do not include any broken circuit as a subset; that is,

$$
P(G, x)=\sum_{\substack{A \subseteq E \\ \forall B \in \mathscr{B}: B \nsubseteq A}}(-1)^{|A|} x^{c(V, A)}
$$

where $\mathscr{B}$ denotes the set of broken circuits of $G$.

As a consequence, since $c(V, A)=|V|-|A|$ whenever $(V, A)$ is cycle-free, the coefficient of $x^{|V|-k}$ in $P(G, x)$ equals $(-1)^{k}$ times the number of $k$-subsets of the edge set of $G$ which do not include any broken circuit of $G$ as a subset $(k=0,1,2, \ldots)$.

The significance of Whitney's broken circuit theorem lies in the fact that it provides a combinatorial interpretation of the coefficients of the chromatic polynomial. It has been generalized to hypergraphs [10, 28], matroids[18], lattices [7, 24], generalized graph colourings [12], and sophisticated inclusion-exclusion variants [11]. In this paper, an even broader generalization is established, from which the aforementioned generalizations derive in a concise and unified way. Some new results are deduced as well, among them a broken circuit theorem for the recent subgraph component polynomial [3, 27], a generalization of the Blass-Sagan theorem on the Möbius function of a finite lattice [7], and a generalization of the well-known maximum-minimums identity [23].

The paper is organized as follows. Section 2 contains the main result along with two different proofs. This main result generalizes Whitney's broken circuit theorem to sums of the type $\sum_{A \subseteq S} f(A)$ where $S$ is a finite set and $f$ is a mapping from the power set of $S$ to an abelian group. In Section 3 conclusions are drawn for the chromatic polynomial of a hypergraph, the subgraph component polynomial and the domination polynomial of a graph, the characteristic polynomial and beta invariant of a matroid, the maximumsminimums-identity, the principle of inclusion-exclusion, the Möbius function of a lattice, the classical Möbius function, Euler's totient function and its Dirichlet inverse, and the reciprocal of the Riemann zeta function. In Section 4 our main result is even further generalized to convex geometries (a concept equivalent to antimatroids). Roughly speaking, 
this generalization states that, if its requirements are fulfilled, the sum $\sum_{A \subseteq S} f(S)$ can be restricted to the free sets of a convex geometry on $S$.

\section{Main result}

Our main result, which is stated below, specializes to Whitney's broken circuit theorem for any finite simple graph $G$ by letting $S$ be the edge set of $G, \mathscr{C}$ the set of all edge sets of cycles of $G, \Gamma=\mathbb{Z}[x]$ with the usual addition of polynomials, $f: 2^{S} \rightarrow \Gamma$ defined by $f(A)=(-1)^{|A|} x^{c(V, A)}$ for any $A \subseteq S$, and $\mathscr{B}=\{C \backslash\{\max C\} \mid C \in \mathscr{C}\}$.

Theorem 1. Let $S$ be a finite linearly ordered set, $\mathscr{C} \subseteq 2^{S} \backslash\{\emptyset\}, \Gamma$ an abelian group (additively written), and $f: 2^{S} \rightarrow \Gamma$ a mapping such that for any $C \in \mathscr{C}$ and $A \supseteq C$,

$$
f(A)+f(A \backslash\{\max C\})=0 .
$$

Then, for any $\mathscr{B} \subseteq\{C \backslash\{\max C\} \mid C \in \mathscr{C}\}$,

$$
\sum_{A \subseteq S} f(A)=\sum_{\substack{A \subseteq S \\ \forall B \in \mathscr{B}: B \nsubseteq A}} f(A)
$$

Subsequently, we give two proofs of Theorem 1. The first proof makes use of the principle of inclusion-exclusion, while the second proof is by induction on $|\mathscr{B}|$.

First Proof. If $\emptyset \in \mathscr{B}$, then $C=\{c\}$ for some $C \in \mathscr{C}$ and $c \in S$, and hence, by the requirement of the theorem, $f(A)+f(A \backslash\{c\})=0$ for any $A \supseteq\{c\}$. It follows that

$$
\sum_{A \subseteq S} f(A)=\sum_{\substack{A \subseteq S \\ A \ni c}} f(A)+\sum_{\substack{A \subseteq S \\ A \not \supset c}} f(A)=\sum_{\substack{A \subseteq S \\ A \ni c}}(f(A)+f(A \backslash\{c\}))=0,
$$

which implies the validity of Eq. (4) since no set $A$ satisfies $\emptyset \nsubseteq A$.

In the sequel, we assume that $\emptyset \notin \mathscr{B}$. By the principle of inclusion-exclusion,

$$
\begin{aligned}
\sum_{\substack{A \subseteq S \\
\forall B \in \mathscr{B}: B \nsubseteq A}} f(A) & =\sum_{\mathscr{A} \subseteq \mathscr{B}}(-1)^{|\mathscr{A}|} \sum_{\substack{A \subseteq S \\
A \supseteq \bigcup \mathscr{A}}} f(A) \\
& =\sum_{A \subseteq S} f(A)+\left[\sum_{\substack{\mathscr{A} \subseteq \mathscr{B} \\
\mathscr{A} \neq \emptyset}}(-1)^{|\mathscr{A}|} \sum_{\substack{A \subseteq S \\
A \supseteq \bigcup \mathscr{A}}} f(A)\right] .
\end{aligned}
$$

It remains to show that the bracketed term in Eq. (5) vanishes, which is the case if

$$
\sum_{A \supseteq \bigcup \mathscr{A}} f(A)=0 \quad(\emptyset \neq \mathscr{A} \subseteq \mathscr{B})
$$


In order to establish Eq. (6), choose (i) $B \in \mathscr{A}$ with $\max B=\max \bigcup \mathscr{A}$, and (ii) $b \notin B$ such that $B \cup\{b\} \in \mathscr{C}$ and $b>\max B$. Then, $b \notin \cup \mathscr{A}$ since otherwise $b \leqslant \max \bigcup \mathscr{A}=$ $\max B$. Hence,

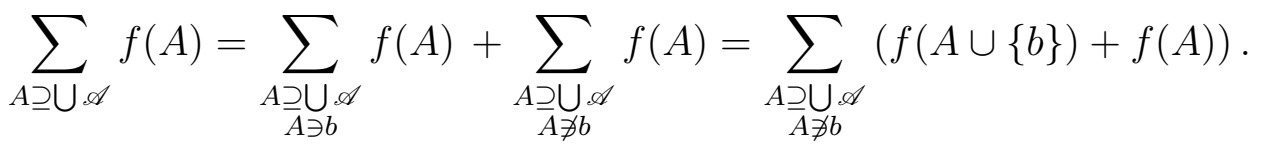

For each $A$ in the last sum consider $A^{\prime}=A \cup\{b\}$. Since $A \supseteq \bigcup \mathscr{A} \supseteq B$ we have $A^{\prime} \supseteq C$ for $C=B \cup\{b\}$ and hence, by the requirement of the theorem,

$$
f(A \cup\{b\})+f(A)=f\left(A^{\prime}\right)+f\left(A^{\prime} \backslash\{\max C\}\right)=0 .
$$

Now Eqs. (7) and (8) imply Eq. (6), and hence the statement of the theorem.

Second Proof. The statement is obvious if $\mathscr{B}=\emptyset$. We proceed by induction on $|\mathscr{B}|$. If $\mathscr{B} \neq \emptyset$, then for some $C \in \mathscr{C}, C \backslash\{\max C\} \in \mathscr{B}$. Among those $C$ choose one whose $\max C$ value is maximal. Let $\mathscr{B}^{\prime}:=\mathscr{B} \backslash\{C \backslash\{\max C\}\}$. By the induction hypothesis,

$$
\sum_{A \subseteq S} f(A)=\sum_{\substack{A \subseteq S \\ \forall B \in \mathscr{B}^{\prime}: B \nsubseteq A}} f(A)
$$

which implies

$$
\sum_{A \subseteq S} f(A)=\sum_{\substack{A \subseteq S \\ \forall B \in \mathscr{B}: B \nsubseteq A}} f(A)+\sum_{\substack{A \subseteq S \\ \forall B \in \mathscr{B}^{\prime}: B \nsubseteq A \\ C \backslash\{\max C\} \subseteq A}} f(A)
$$

We claim that the second sum on the right-hand side of Eq. (9) vanishes. Let

$$
\mathscr{A}:=\left\{A \mid A \subseteq S ; \forall B \in \mathscr{B}^{\prime}: B \nsubseteq A ; C \backslash\{\max C\} \subseteq A\right\} .
$$

By Eq. (3) our claim is proved if $A \in \mathscr{A}$ if and only if $A \backslash\{\max C\} \in \mathscr{A}$. The only non-trivial issue is to show that if $A \backslash\{\max C\} \in \mathscr{A}$, then $B \nsubseteq A$ for any $B \in \mathscr{B}^{\prime}$. Assume that $B \subseteq A$ for some $B \in \mathscr{B}^{\prime}$. By the requirement of the theorem there exists $C^{\prime} \in \mathscr{C}$ such that $B=C^{\prime} \backslash\left\{\max C^{\prime}\right\}$. If $\max C \in B$, then $\max C \leqslant \max B<\max C^{\prime}$, contradicting the maximality of $\max C$. If $\max C \notin B$, then since $A \supseteq B$ we conclude that $A \backslash\{\max C\} \supseteq B$, which is in contradiction with $A \backslash\{\max C\} \in \mathscr{A}$.

Remark 1. Let $S$ be a finite poset, $\mathscr{C}=\{\{s, t\} \subseteq S \mid s<t\}$, and $f$ a mapping satisfying the requirement in Eq. (3) with respect to some linear extension of $S$. By Theorem 1,

$$
\sum_{A \subseteq S} f(A)=\sum_{A \subseteq S_{\max }} f(A)
$$

where $S_{\max }$ denotes the set of maximal elements in $S$. 
Remark 2. If $S$ is an upper semilattice, we may choose $\mathscr{C}=\{\{s, t, s \vee t\} \mid s \| t\}$, where $s \| t$ signifies that $s$ and $t$ are incomparable and $s \vee t$ denotes the least upper bound of $s$ and $t$ in $S$. Thus, for any mapping $f$ satisfying the requirement in Eq. (3) we have

$$
\sum_{A \subseteq S} f(A)=\sum_{\substack{A \subseteq S \\ A \text { chain }}} f(A) .
$$

We will make use of the preceding two identities in Subsection 3.8.

\section{Applications}

\subsection{Chromatic polynomial of a hypergraph}

A hypergraph is a pair $H=(V, \mathscr{E})$ where $V$ is a set (of vertices) and $\mathscr{E}$ is a set of non-empty subsets of $V$ (called edges). $H$ is called finite if $V$ is finite, and simple if $|E| \geqslant 2$ for any $E \in \mathscr{E}$. Distinct vertices $v, w \in V$ such that $v, w \in E$ for some $E \in \mathscr{E}$ are called adjacent. The reflexive and transitive closure of the adjacency relation yields an equivalence relation on $V$, whose equivalence classes are referred to as connected components of $H$, and whose number of equivalence classes is denoted by $c(H)$.

By applying the principle of inclusion-exclusion it follows that for any finite simple hypergraph $H$ and any $x \in \mathbb{N}$ the polynomial

$$
P(H, x)=\sum_{A \subseteq \mathscr{E}}(-1)^{|A|} x^{c(V, A)}
$$

evaluates to the number of mappings $f: V \rightarrow\{1, \ldots, x\}$ such that $\left.f\right|_{E}$ (the restriction of $f$ to $E$ ) is non-constant for any $E \in \mathscr{E}$ (see [10] for details).

We consider cycles in hypergraphs in the classical sense of C. Berge [4]. Accordingly, a cycle of length $l$ in a hypergraph $H$ is any finite sequence $\left(v_{1}, E_{1}, v_{2}, E_{2}, \ldots, v_{l}, E_{l}, v_{l+1}\right)$ consisting of at least two pairwise distinct vertices $v_{1}, \ldots, v_{l} \in V$ resp. edges $E_{1}, \ldots, E_{l} \in$ $\mathscr{E}$ where $v_{1}=v_{l+1}$ and $v_{i}, v_{i+1} \in E_{i}$ for $i=1, \ldots, l$. The definition of a broken circuit is similar as for graphs: Given a linear ordering relation on $\mathscr{E}$, for any set $C$ consisting of the edges of a cycle of $H$ we refer to $C \backslash\{\max C\}$ as a broken circuit of $H$.

Let $H=(V, \mathscr{E})$ be a finite simple hypergraph whose edge set is endowed with a linear ordering relation. In order to apply Theorem 1 we choose $S=\mathscr{E}, \mathscr{C}$ as a set of edge sets of cycles of $H, \Gamma=\mathbb{Z}[x]$ with the usual addition of polynomials, $f(A)=(-1)^{|A|} x^{c(V, A)}$ for any subsets $A \subseteq S$, and $\mathscr{B} \subseteq\{C \backslash\{\max C\} \mid C \in \mathscr{C}\}$. This gives

$$
P(H, x)=\sum_{\substack{A \subseteq \mathscr{E} \\ \forall B \in \mathscr{B}: B \nsubseteq A}}(-1)^{|A|} x^{c(V, A)}
$$

provided, of course, that the requirement in Eq. (3) is satisfied for any $C \in \mathscr{C}$ and any $A \supseteq C$. This can be guaranteed by imposing one of the following requirements on $\mathscr{C}$ : 
(a) All cycles belonging to $\mathscr{C}$ have the property that each edge of the cycle is included by the union of the other edges of that cycle.

(b) All cycles belonging to $\mathscr{C}$ contain an edge of cardinality 2 , and these edges constitute an upset of the edge set with respect to the given linear ordering relation.

When applied to (a), Theorem 1 from Section 2 provides us with a new proof of [28, Theorem 8]. When applied to (b), it leads to a new proof of [10, Theorem 2].

The requirement in (a) is satisfied if $\mathscr{C}$ arises from cycles in $H$ having the property that each edge on the cycle is included by the union of its two neighbouring edges. This latter condition holds, e.g., for $l$-tight cycles in $r$-uniform hypergraphs where $l \geqslant r / 2$; these are cycles "whose vertices can be cyclically ordered in such a way that the edges are segments of this ordering and every two consecutive edges intersect in exactly $l$ vertices" [16]. Recall that a hypergraph is referred to as $r$-uniform if each edge contains exactly $r$ vertices. An $(r-1)$-tight cycle in an $r$-uniform hypergraph is called tight. Thus, choosing $\mathscr{C}$ from the tight cycles of an $r$-uniform hypergraph satisfies (a).

As a more concrete example for (a), consider the 4-uniform hypergraph $H=(V, \mathscr{E})$ on the set of lattice points of a finite rectangular grid where the edges of $H$ are any four points determining a rectangle. Let $\mathscr{C}$ be the set of all 3 -sets of edges arising from the 2-tight cycles of length three in $H$. Since any 2-tight cycle of length three corresponds to a pair of neighbouring rectangles (that is, rectangles having two points in common, thus determining another, geometrically larger rectangle) we can order the edges of $H$ in such a way that edges corresponding to geometrically larger rectangles occur later in the ordering. In this way, the sum in Eq. (12) can be restricted to those subsets $A$ of $\mathscr{E}$ that contain no neighbouring rectangles. Rectangle-free grid colorings are a topic of active research; see e.g., [26] for recent results.

\subsection{Subgraph component polynomial}

Introduced by Averbouch, Makowsky, and Tittmann [3, 27], the subgraph component polynomial of any finite graph $G=(V, E)$ is defined by

$$
Q(G, x, y)=\sum_{A \subseteq V} x^{|A|} y^{c(G[A])}
$$

This polynomial has seen applications in social network analysis [3] and formal language theory [6]. For some recent results on $Q(G, x, y)$, the reader is referred to [20].

In the following, our considerations are restricted to the particular case where $x=-1$. We refer to $G$ as cyclically claw-free if no centre of a claw is located on a cycle. Evidently, any claw-free or cycle-free graph is cyclically claw-free.

The key observation is that if $G$ is cyclically claw-free, then $c(G[A])=c(G[A \backslash\{c\}])$ for any $A \subseteq V$ and any vertex $c$ on a cycle $C \subseteq A$ (where, in this subsection, we consider cycles as subsets of the vertex set). This leads to a vertex analogue of the notion of a broken circuit: Given a linear ordering relation on $V$, for any cycle $C \subseteq V$ we refer to 
$C \backslash\{\max C\}$ as a broken circuit of $G$. Similar to Eq. (12) we obtain by Theorem 1,

$$
Q(G,-1, y)=\sum_{\substack{A \subseteq V \\ \forall B \in \mathscr{B}: B \nsubseteq A}}(-1)^{|A|} y^{c(G[A])}
$$

for any cyclically claw-free finite graph $G$ and any set $\mathscr{B} \subseteq 2^{V}$ of broken circuits of $G$. Note that if we choose $\mathscr{B}$ as the set of all broken circuits of $G$, then any subset $A$ of $V$ in the preceding sum is cycle-free, and hence satisfies $c(G[A])=|A|-m(G[A])$. Thus, we obtain

$$
Q(G,-1, y)=\sum_{A}(-1)^{|A|} y^{|A|-m(G[A])}
$$

where the sum extends over all subsets $A$ of $V$ not including any broken circuit. As a consequence, $Q(G,-1,-1)$ is the number of broken-circuit-free vertex-induced subgraphs having an even number of edges minus those having an odd number of edges.

We finally remark that Eqs. (13) and (14) hold with $x$ in place of -1 if $Q(G, x, y)$ is considered as a polynomial over some commutative ring where $(x+1) y=0$.

\subsection{Domination polynomial}

The domination polynomial of any finite simple graph $G=(V, E)$, introduced by Arocha and Llano [2], is the generating function

$$
D(G, x):=\sum_{k=0}^{|V|} d_{k}(G) x^{k}
$$

where $d_{k}(G)$ is the number of $k$-subsets $A$ of $V$ satisfying $N_{G}[A]=V$. Here, $N_{G}[A]$ denotes the closed neighbourhood of $A$ in $G$, that is, the union of $A$ and its set of neighbours in $G$. For convenience, we write $N_{G}[v]$ in place of $N_{G}[\{v\}]$ for any $v \in V$.

Given a linear ordering relation on the vertex set of $G$, for any $v \in V$ we refer to $N_{G}[v] \backslash\{v\}$ as a broken neighbourhood of $G$ if $v=\max N_{G}[v]$. In [13] it is shown that

$$
D(G, x)=\sum_{A \subseteq V}(-1)^{|A|}(x+1)^{|V|-\left|N_{G}[A]\right|},
$$

and moreover, if $G$ does not have isolated vertices, then this sum can be restricted to those subsets $A$ of $V$ which do not include any broken neighbourhoods from an arbitrary set of broken neighbourhoods of $G$.

This latter statement easily derives from our main result in Section 2 and Eq. (15) by considering the mapping $f(A)=(-1)^{|A|}(x+1)^{|V|-\left|N_{G}[A]\right|}$ for any $A \subseteq V$ and letting $\mathscr{C}$ be the set of all closed neighbourhoods $N_{G}[v]$ where $v=\max N_{G}[v]$. The requirement in Eq. (3) is satisfied since $N_{G}[A \backslash\{v\}]=N_{G}[A]$ for any $v \in V$ and any $A \supseteq N_{G}[v]$.

As noted in [13], if $G$ does not have isolated vertices or isolated edges, and its vertex set is linearly ordered such that the vertices of degree 1 constitute an upset, then each pendant edge $\{v, w\}$ where $v$ is of degree 1 gives rise to a broken neighbourhood $\{w\}$. In this case, the sum in Eq. (15) can be restricted to those subsets $A$ of $V$ which do not contain any vertex from a set of vertices which are adjacent to a vertex of degree 1 . 


\subsection{Characteristic polynomial and beta invariant}

Similar conclusions as for graph and hypergraph polynomials can be drawn for the characteristic polynomial [18] and the beta invariant [8] of a matroid.

Recall that a matroid is a pair $M=(E, r)$ consisting of a finite set $E$ and a $\mathbb{Z}$-valued function $r$ on $2^{E}$ such that for any $A, B \subseteq E$,

(i) $0 \leqslant r(A) \leqslant|A|$,

(ii) $A \subseteq B \Rightarrow r(A) \leqslant r(B)$,

(iii) $r(A \cup B)+r(A \cap B) \leqslant r(A)+r(B)$.

A circuit of $M$ is a non-empty subset $C \subseteq E$ such that $r(C \backslash\{c\})=|C|-1=r(C)$ for any $c \in C$. Given a linear ordering relation on $E$, for any circuit $C$ of $M$ we refer to $C \backslash\{\max C\}$ as a broken circuit of $M$.

The characteristic polynomial $\chi(M, x)$ and the beta invariant $\beta(M)$ of a matroid $M=(E, r)$ are defined by

$$
\begin{aligned}
& \chi(M, x)=\sum_{A \subseteq E}(-1)^{|A|} x^{r(E)-r(A)}, \\
& \beta(M)=(-1)^{r(E)} \sum_{A \subseteq E}(-1)^{|A|} r(A) .
\end{aligned}
$$

In order to apply Theorem 1 , let $f_{1}: 2^{E} \rightarrow \mathbb{Z}[x]$ be defined by $f_{1}(A)=(-1)^{|A|} x^{r(E)-r(A)}$, and $f_{2}: 2^{E} \rightarrow \mathbb{Z}$ by $f_{2}(A)=(-1)^{|A|} r(A)$. Let $\mathscr{C}$ denote the set of all circuits of $M$. Then, for any $C \in \mathscr{C}$ and any $A \supseteq C, r(A \backslash\{\max C\})=r(A)$; hence, both $f_{1}$ and $f_{2}$ satisfy the requirement in Eq. (3). Let $\mathscr{B}$ denote the set of broken circuits of $M$. Then, by Theorem 1, the sums in Eqs. (16) and (17) can be restricted to those subsets $A$ of $E$ not including any $B \in \mathscr{B}$ as a subset. No such $A$ may include a circuit, since otherwise it would include the broken circuit derived from it. Therefore, $r(A)=|A|$ and hence,

$$
\begin{gathered}
\chi(M, x)=\sum_{\substack{A \subseteq E \\
\forall B \in \mathscr{\mathscr { B }}: B \nsubseteq A}}(-1)^{|A|} x^{r(E)-r(A)}=\sum_{k=0}^{|E|}(-1)^{k} b_{k}(M) x^{r(E)-k}, \\
\beta(M)=(-1)^{r(E)} \sum_{\substack{A \subseteq E \\
\forall B \in \mathscr{B}: B \nsubseteq A}}(-1)^{|A|} r(A)=(-1)^{r(E)} \sum_{k=1}^{|E|}(-1)^{k} k b_{k}(M)
\end{gathered}
$$

where $b_{k}(M)$ denotes the number of $k$-subsets of $E$ including no broken circuit.

Eq. (19) can alternatively be deduced from Eq. (18), which is due to Heron [18], by considering the derivative of $\chi(M, x)$ at $x=1$. 


\subsection{Maximum-minimums identity}

Let $\Gamma$ be an abelian group, endowed with a linear ordering relation, $\left(x_{s} \mid s \in S\right)$ a finite family of elements from $\Gamma, k \in \mathbb{N}$, and $f: 2^{S} \rightarrow \Gamma$ defined by

$$
f(A)= \begin{cases}(-1)^{|A|-k} \min _{k}\left(x_{a} \mid a \in A\right), & \text { if }|A| \geqslant k \\ 0, & \text { if }|A|<k\end{cases}
$$

where $\min _{k}\left(x_{a} \mid a \in A\right)$ denotes the $k$-th smallest element in $\left(x_{a} \mid a \in A\right)$ for any $A \subseteq S$ satisfying $|A| \geqslant k$.

In order to define $\mathscr{B}$ and $\mathscr{C}$, choose some linear ordering relation on $S$ such that $s<t$ implies $x_{s} \leqslant x_{t}$ for any $s, t \in S$. Now, define $\mathscr{B}=\{C \backslash\{\max C\} \mid C \in \mathscr{C}\}$ where $\mathscr{C}$ is the set of all $(k+1)$-subsets of $S$. Evidently, for any $C \in \mathscr{C}$ and any $A \supseteq C$,

$$
\min _{k}\left(x_{a} \mid a \in A \backslash\{\max C\}\right)=\min _{k}\left(x_{a} \mid a \in A\right) .
$$

Hence, the requirements of Theorem 1 are satisfied, which gives

$$
\sum_{\substack{A \subseteq S \\|A| \geqslant k}}(-1)^{|A|-k} \min _{k}\left(x_{a} \mid a \in A\right)=\sum_{\substack{A \subseteq S \\|A| \geqslant k \\ \forall B \in \mathscr{B}: B \nsubseteq A}}(-1)^{|A|-k} \min _{k}\left(x_{a} \mid a \in A\right) .
$$

Since $\mathscr{B}$ consists of all $k$-subsets of $S \backslash\{\max S\}$, the last two conditions under the second sum in Eq. (20) are equivalent to $|A|=k$ and $\max S \in A$. Since there are $\left(\begin{array}{c}|S|-1 \\ k-1\end{array}\right)$ many such $A$, and each of them satisfies $\min _{k}\left(x_{a} \mid a \in A\right)=\max \left(x_{s} \mid s \in S\right)$, we find that

$$
\sum_{\substack{A \subseteq S \\
|A| \geqslant k}}(-1)^{|A|-k} \min _{k}\left(x_{a} \mid a \in A\right)=\left(\begin{array}{c}
|S|-1 \\
k-1
\end{array}\right) \max \left(x_{s} \mid s \in S\right) .
$$

Note that neither side of this identity depends on the ordering of $S$. For $k=1$ this identity is known as the maximum-minimums identity; see [23] for a probabilistic proof (in case that the $x_{s}$ 's are reals) and an application to the coupon collector problem.

\subsection{Principle of inclusion-exclusion}

Let $\left\{M_{s}\right\}_{s \in S}$ be a finite family of finite sets, where $S$ is linearly ordered, $\mathscr{B}$ a set of nonempty subsets of $S$ such that for any $B \in \mathscr{B}, \bigcap_{b \in B} M_{b} \subseteq M_{c}$ for some $c=c(B)>\max B$. In [11] it is shown that

$$
\left|\bigcup_{s \in S} M_{s}\right|=\sum_{\substack{\emptyset \neq A \subseteq S \\ \forall B \in \mathscr{B}: B \nsubseteq A}}(-1)^{|A|-1}\left|\bigcap_{a \in A} M_{a}\right|
$$


Under the above assumptions, this identity (which has applications to network and system reliability) follows from Theorem 1 by defining $\mathscr{C}=\{B \cup c(B) \mid B \in \mathscr{B}\}$ and

$$
f(A)= \begin{cases}(-1)^{|A|-1}\left|\bigcap_{a \in A} M_{a}\right|, & \text { if } A \neq \emptyset, \\ 0, & \text { if } A=\emptyset,\end{cases}
$$

and applying the principle of inclusion-exclusion to the sets $M_{s}, s \in S$.

A particular case of Eq. (22) is Narushima's principle of inclusion-exclusion [22] where the sum extends over all chains of a semilattice:

$$
\left|\bigcup_{s \in S} M_{s}\right|=\sum_{\substack{\emptyset \neq A \subseteq S \\ A \text { chain }}}(-1)^{|A|-1}\left|\bigcap_{a \in A} M_{a}\right|
$$

As a prerequisite, $(S, \vee)$ is required to be a finite upper semilattice satisfying $M_{s} \cap M_{t} \subseteq$ $M_{s \vee t}$ for any $s, t \in S$. This particular case of Eq. (22) may also be deduced from Eq. (11).

\subsection{Möbius function of a lattice}

Our next application concerns the Möbius function of a finite lattice. For notions from the theory of partially ordered sets and lattices, we refer to the textbook of Graetzer [17].

Recall that the Möbius function of any finite lattice $L=[\hat{0}, \hat{1}]$ is the unique $\mathbb{Z}$-valued function $\mu_{L}: L \rightarrow \mathbb{Z}$ such that for any $x \in L$,

$$
\sum_{y \leqslant x} \mu_{L}(y)=\delta_{\hat{0} x}
$$

where $\delta$ denotes the Kronecker delta. Following Rota [24], we write $\mu(L)$ instead of $\mu_{L}(\hat{1})$ and introduce the notion of a crosscut, which is any antichain $C \subseteq L \backslash\{\hat{0}, \hat{1}\}$ having a non-empty intersection with any maximal chain from $\hat{0}$ to $\hat{1}$ in $L$. As a prerequisite, $L$ must be non-trivial, that is, $L \backslash\{\hat{0}, \hat{1}\} \neq \emptyset$. Rota's crosscut theorem [24] states that for any non-trivial finite lattice $L=[\hat{0}, \hat{1}]$ and any crosscut $C$ of $L$,

$$
\mu(L)=\sum_{\substack{A \subseteq C \\ \wedge A=\hat{0}, \bigvee A=\hat{1}}}(-1)^{|A|}
$$

where $\bigwedge \emptyset=\hat{1}$ and $\bigvee \emptyset=\hat{0}$. Due to Blass and Sagan [7], for $C=A(L)$, which is the crosscut of all atoms of $L$, this sum can be written as ${ }^{1}$

$$
\mu(L)=\sum_{\substack { A \subseteq C \\
\begin{subarray}{c}{A=0, \bigvee \\
\forall B \in \hat{B}{ A \subseteq C \\
\begin{subarray} { c } { A = 0 , \bigvee \\
\forall B \in \hat { B } } }\end{subarray}}(-1)^{|A|}
$$

\footnotetext{
${ }^{1}$ For $C=A(L)$ the conditions $\bigwedge A=\hat{0}$ in Eq. (25) and $\bigwedge B<c$ in Eq. (26) can be omitted.
} 
where, according to some fixed partial ordering relation $\unlhd$ on $C, \mathscr{B}$ consists of all nonempty subsets $B$ of $C$ such that for any $b \in B$ there is some $c=c(B, b) \in C$ satisfying

$$
c \triangleleft b \text { and } \bigwedge B<c<\bigvee B
$$

Here and subsequently, $<, \wedge$ and $\vee$ are associated with the lattice ordering $\leqslant$ in $L$, while $\triangleleft$ is associated with the additional partial ordering relation $\unlhd$ on $C$.

Blass and Sagan [7] used their result in computing and combinatorially explaining the Möbius function of various lattices and in generalizing Stanley's well-known theorem [25] that the characteristic polynomial of a semimodular supersolvable lattice factors over the integers. As noted by Blass and Sagan [7], for $C=A(L)$ Eq. (25) generalizes Eq. (24), which is easily seen by considering the total incomparability $\unlhd$ order on $C$.

We now prove that Eq. (25) holds for any crosscut $C$ of $L$ by applying our main result from Section 2 in dual form to the sum in Eq. (24). Thus, we consider $f: 2^{C} \rightarrow \mathbb{Z}$ where

$$
f(A):= \begin{cases}(-1)^{|A|} & \text { if } \bigwedge A=\hat{0} \text { and } \bigvee A=\hat{1}, \\ 0 & \text { otherwise, }\end{cases}
$$

for any $A \subseteq C$. According to some arbitrary linear extension of $\unlhd$ on $C$ define

$$
\mathscr{C}:=\left\{B \cup\left\{\min _{b \in B} c(B, b)\right\} \mid B \in \mathscr{B}\right\},
$$

which implies $\mathscr{B}=\left\{C^{\prime} \backslash\left\{\min C^{\prime}\right\} \mid C^{\prime} \in \mathscr{C}\right\}$. It remains to check that for any $C^{\prime} \in \mathscr{C}$ and any $A \supseteq C^{\prime}$ the requirement in Eq. (3) holds. To this end, we show that

$$
\bigwedge A=\bigwedge\left(A \backslash\left\{\min C^{\prime}\right\}\right), \bigvee A=\bigvee\left(A \backslash\left\{\min C^{\prime}\right\}\right)
$$

For the first identity in (27), choose $B \in \mathscr{B}$ such that $C^{\prime}=B \cup\left\{\min _{b \in B} c(B, b)\right\}$. Then,

$$
\begin{aligned}
\bigwedge A & =\bigwedge\left(A \backslash C^{\prime}\right) \wedge \bigwedge C^{\prime} \quad\left(\text { since } A \supseteq C^{\prime}\right) \\
& =\bigwedge\left(A \backslash\left(B \cup\left\{\min _{b \in B} c(B, b)\right\}\right)\right) \wedge \bigwedge\left(B \cup\left\{\min _{b \in B} c(B, b)\right\}\right) \\
& =\bigwedge\left(A \backslash\left(B \cup\left\{\min _{b \in B} c(B, b)\right\}\right)\right) \wedge \bigwedge B \quad\left(\text { since } \min _{b \in B} c(B, b)>\bigwedge B\right) \\
& =\bigwedge\left(A \backslash\left\{\min _{b \in B} c(B, b)\right\}\right) \quad\left(\text { since } A \supseteq B \text { and } \min _{b \in B} c(B, b) \notin B\right) \\
& =\bigwedge\left(A \backslash\left\{\min C^{\prime}\right\}\right) \quad\left(\text { since } \min C^{\prime}=\min _{b \in B} c(B, b)\right) .
\end{aligned}
$$

For the second claim in Eq. (27), simply exchange $\wedge$ with $\vee$ and $>$ with $<$. Thus, for any crosscut $C$ of $L$, the identity in Eq. (25) follows from our main result in Section 2. Furthermore, the identity remains valid if $\mathscr{B}$ is replaced by any subset $\mathscr{B}^{\prime} \subseteq \mathscr{B}$.

\subsection{Arithmetical functions}

In this subsection, we establish new gcd- and lcm-sum expansions for some classical arithmetical functions. We refer to the textbook of Apostol [1] for a comprehensive account of arithmetical functions in general, and of multiplicative functions in particular. 


\subsubsection{The classical Möbius function}

For any $n \in \mathbb{N}$ let $L_{n}$ denote the lattice of positive divisors of $n$, and $\mu(n)=\mu\left(L_{n}\right)$ the classical Möbius function of $n$. It is well-known that for $n \geqslant 1$ and $k \geqslant 0$,

$$
\mu(n)= \begin{cases}(-1)^{k}, & \text { if } n \text { is the product of } k \text { distinct primes, } \\ 0, & \text { otherwise. }\end{cases}
$$

We use $\operatorname{gcd}(A)$ and $\operatorname{lcm}(A)$ to denote the greatest common divisor resp. least common multiple of any finite set $A \subseteq \mathbb{N}$. We adopt the convention that $\operatorname{gcd}(\emptyset)=0$ and $\operatorname{lcm}(\emptyset)=1$. With $f(A)=(-1)^{|A|}$ if $\operatorname{gcd}(A)=1$ and $f(A)=0$ if $\operatorname{gcd}(A)>1$ for $A \subseteq L_{n} \backslash\{1, n\}$ the first remark after Theorem 1 implies that for any non-prime $n>1$,

$$
\sum_{\substack{A \subseteq L_{n} \backslash\{1, n\} \\ \operatorname{gcd}(A)=1}}(-1)^{|A|}=\sum_{\substack{A \subseteq P_{n}^{*} \\ \operatorname{gcd}(A)=1}}(-1)^{|A|}=\sum_{\substack{A \subseteq P_{n} \\ \operatorname{gcd}\left(A^{*}\right)=1}}(-1)^{|A|}=\sum_{\substack{A \subseteq P_{n} \\ \operatorname{lcm}(A)=n}}(-1)^{|A|}=\mu(n)
$$

where $P_{n}$ denotes the set of prime factors of $n$, and $A^{*}=\left\{\frac{n}{a} \mid a \in A\right\}$ for any $A \subseteq P_{n}$. The first equality in Eq. (29) is due to Eq. (10), while the last one follows from Eq. (28) (or Eq. (24) with $C=P_{n}$ ). Similarly, by considering the dual order on $L_{n}$ we obtain

$$
\sum_{\substack{A \subseteq L_{n} \backslash\{1, n\} \\ \operatorname{lcm}(A)=n}}(-1)^{|A|}=\sum_{\substack{A \subseteq P_{n} \\ \operatorname{lcm}(A)=n}}(-1)^{|A|}=\sum_{\substack{A \subseteq P_{n} \\ \operatorname{gcd}\left(A^{*}\right)=1}}(-1)^{|A|}=\sum_{\substack{A \subseteq P_{n}^{*} \\ \operatorname{gcd}(A)=1}}(-1)^{|A|}=\mu(n)
$$

for each non-prime integer $n \geqslant 1$. For $n>1$, the requirement that $n$ is non-prime is necessary in order to ensure that $P_{n}, P_{n}^{*} \subseteq L_{n} \backslash\{1, n\}$ in Eqs. (29) and (30). It can be omitted by considering $L_{n} \backslash\{n\}$ in Eq. (29) and $L_{n} \backslash\{1\}$ in Eq. (30), respectively.

If $n$ is not squarefree, then $\mu(n)=0$ and hence, due to Eqs. (29) and (30), the abstract simplicial complexes

$$
\begin{aligned}
& \mathscr{S}_{n}=\left\{A \subseteq L_{n} \backslash\{1, n\} \mid \operatorname{gcd}(A)>1\right\}, \\
& \mathscr{T}_{n}=\left\{A \subseteq L_{n} \backslash\{1, n\} \mid A \neq \emptyset \text { and } \operatorname{lcm}(A)<n\right\}
\end{aligned}
$$

have Euler characteristic 1. Recall that the Euler characteristic $\chi(\mathscr{A})$ of an abstract simplicial complex $\mathscr{A}$ is defined as $\chi(\mathscr{A})=\sum_{A \in \mathscr{A}}(-1)^{|A|-1}$, and that $\mathscr{A}$ is called contractible if its geometric realization as a simplicial complex is contractible, which means, roughly speaking, that it can be continuously shrunk to a point. It is well-known that if $\mathscr{A}$ is contractible, then $\chi(\mathscr{A})=1$. In view of this, one might conjecture that both $\mathscr{S}_{n}$ and $\mathscr{T}_{n}$ are contractible if $n$ is not squarefree. This is indeed the case: Suppose $p^{2} \mid n$ for some prime $p$. For any $A \in \mathscr{S}_{n}$, if $\operatorname{gcd}(A) \mid \frac{n}{p}$, then $\operatorname{gcd}\left(A \cup\left\{\frac{n}{p}\right\}\right)=\operatorname{gcd}(A)>1$; if $\operatorname{gcd}(A) \nmid \frac{n}{p}$, then $p^{2} \mid \operatorname{gcd}(A)$ and hence, $\operatorname{gcd}\left(A \cup\left\{\frac{n}{p}\right\}\right) \geqslant p>1$. In both cases, $A \cup\left\{\frac{n}{p}\right\} \in \mathscr{S}_{n}$. Thus, $\frac{n}{p}$ is contained in every maximal face of $\mathscr{S}_{n}$. As a consequence, the geometric realization of $\mathscr{S}_{n}$ is star-shaped with respect to $\frac{n}{p}$ and hence contractible. Similarly, by distinguishing the cases $p \mid \operatorname{lcm}(A)$ and $p \nmid \operatorname{lcm}(A)$ we may conclude that $\mathscr{T}_{n}$ is contractible. In fact, $\mathscr{S}_{n}$ and $\mathscr{T}_{n}$ are isomorphic by virtue of $A \mapsto A^{*}$. 
The preceding contractibility result establishes a link to the theory of discrete tubes. Due to Corollary 2 of [21], for any contractible abstract simplicial complex $\mathscr{A}$,

$$
(-1)^{r} \sum_{\substack{A \in \mathscr{A} \\|A| \leqslant r}}(-1)^{|A|-1} \leqslant(-1)^{r} \quad(r=1,2,3, \ldots) .
$$

Applying this to $\mathscr{A}=\mathscr{S}_{n}$ resp. $\mathscr{T}_{n}$ gives Bonferroni-like inequalities on gcd- and lcm-sums, e.g., in Eqs. (29) and (30), in the particular case where $n$ is non-squarefree.

\subsubsection{Euler's totient function}

Our conclusions on Euler's totient function and its Dirichlet inverse (cf. Subsection 3.8.3) are stated more generally using the notion of a multiplicative function. We refer to any function $h: \mathbb{N} \rightarrow \mathbb{C}$ as multiplicative if $h(1)=1$ and $h(a b)=h(a) h(b)$ for any coprime $a, b \in \mathbb{N}$, and as completely multiplicative if the latter condition holds for any $a, b \in \mathbb{N}$.

Examples of multiplicative functions are the identity function, the power functions for any complex exponent, and the Liouville function, which are all completely multiplicative; further examples include the Möbius function, Euler's totient function, and the sum of positive divisors of $n$.

Let $f(A)=(-1)^{|A|-1} h(\operatorname{gcd}(A))$ for $A \subseteq L_{n} \backslash\{1, n\}$ where $h$ is multiplicative and non-vanishing on the set of primes. Then, $h(n) \neq 0$ for $n \in \mathbb{N}$-provided $n$ is squarefree or $h$ is completely multiplicative. In both cases, $h(d) \neq 0$ and $h(n / d)=h(n) / h(d)$ for any positive divisor $d$ of $n$. In view of this, the first remark following Theorem 1 implies that for any non-prime integer $n \geqslant 1$,

$$
\begin{array}{r}
\sum_{\substack{A \subseteq L_{n} \backslash\{1, n\} \\
A \neq \emptyset}}(-1)^{|A|-1} h(\operatorname{gcd}(A))=\sum_{\substack{A \subseteq P_{n}^{*} \\
A \neq \emptyset}}(-1)^{|A|-1} h(\operatorname{gcd}(A))=\sum_{\substack{A \subseteq P_{n} \\
A \neq \emptyset}}(-1)^{|A|-1} h\left(\operatorname{gcd}\left(A^{*}\right)\right) \\
=\sum_{\substack{A \subseteq P_{n} \\
A \neq \emptyset}}(-1)^{|A|-1} h\left(n \prod_{a \in A} \frac{1}{a}\right)=h(n)-h(n) \sum_{A \subseteq P_{n}}(-1)^{|A|} \prod_{a \in A} \frac{1}{h(a)}
\end{array}
$$

and hence,

$$
\sum_{\substack{A \subseteq L_{n} \backslash\{1, n\} \\ A \neq \emptyset}}(-1)^{|A|-1} h(\operatorname{gcd}(A))=h(n)-h(n) \prod_{\substack{p \mid n \\ p \text { prime }}}\left(1-\frac{1}{h(p)}\right)=: h(n)-\varphi_{h}(n)
$$

provided $n$ is squarefree or $h$ is completely multiplicative. For $h=\operatorname{id}_{\mathbb{N}}$ the function $\varphi_{h}$ in Eq. (33) is known as Euler's totient function, which for any $n \in \mathbb{N}$ evaluates to the number of positive integers coprime with $n$ (sequence A00010 in [19]).

In a similar way to Subsection 3.8.1, the requirement that $n$ is non-prime can be dropped by considering $L_{n} \backslash\{n\}$ instead of $L_{n} \backslash\{1, n\}$ in Eqs. (32) and (33). Furthermore, if $n$ is not squarefree and $h$ completely multiplicative, then $\mu(n)=0$ and hence by Eq. (29) the sum in Eq. (33) (even with the previous modification) can be restricted to $\operatorname{gcd}(A)>1$. 
Since $L_{n} \backslash\{n\}$ is a lower semilattice for any $n \in \mathbb{N}$, we obtain by Eqs. (11) and (33),

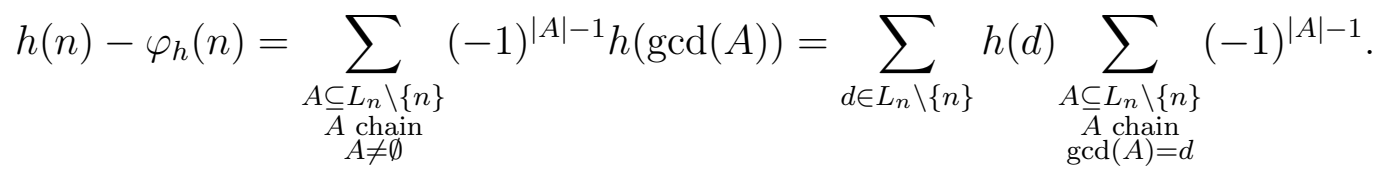

By backward induction on the height of $d$ in $L_{n} \backslash\{n\}$ it can be shown that the inner sum in Eq. (34) agrees with $-\mu(n / d)$. As a consequence,

$$
\varphi_{h}(n)=\sum_{d \mid n} h(d) \mu\left(\frac{n}{d}\right)=h(n) \sum_{d \mid n} \frac{\mu(d)}{h(d)}
$$

provided $n$ is squarefree or $h$ is completely multiplicative. Under this requirement, we rediscover the known formula (cf. Subsection 3.8.3)

$$
\prod_{\substack{p \mid n \\ p \text { prime }}}\left(1-\frac{1}{h(p)}\right)=\sum_{d \mid n} \frac{\mu(d)}{h(d)}
$$

as an immediate consequence of Eq. (35). Eq. (36) also holds for non-squarefree numbers $n$ and any multiplicative function $h$ if $h$ is required to be nowhere zero, or if the sum in Eq. (36) is restricted to $d \mid n$ where $d$ is squarefree. Both modifications immediately follow by applying Eq. (36) to the squarefree kernel of $n$.

\subsubsection{Dirichlet inverse of Euler's totient function}

The dual of Eq. (10), applied to $f(A)=(-1)^{|A|} h(\operatorname{lcm}(A))$ for any $A \subseteq L_{n} \backslash\{1, n\}$ where $h$ is multiplicative reveals that for any non-prime integer $n \geqslant 1$,

$$
\sum_{A \subseteq L_{n} \backslash\{1, n\}}(-1)^{|A|} h(\operatorname{lcm}(A))=\sum_{A \subseteq P_{n}}(-1)^{|A|} \prod_{a \in A} h(a)=\prod_{\substack{p \mid n \\ p \text { prime }}}(1-h(p)) .
$$

For $h=\mathrm{id}_{\mathbb{N}}$ the product on the right-hand side of Eq. (37) is known as the Dirichlet inverse of Euler's totient function (sequence A023900 in [19]). Similar to our discussion on the totient function, the requirement that $n$ is non-prime can be removed by considering $L_{n} \backslash\{1\}$ instead of $L_{n} \backslash\{1, n\}$ in Eq. (37). Furthermore, if $n$ is not squarefree, then $\mu(n)=0$ and hence by Eq. (30), the sum in Eq. (37) can be restricted to $\operatorname{lcm}(A)<n$. Since $L_{n} \backslash\{1\}$ is an upper semilattice for any $n \in \mathbb{N}$, we obtain by Eqs. (11) and (37),

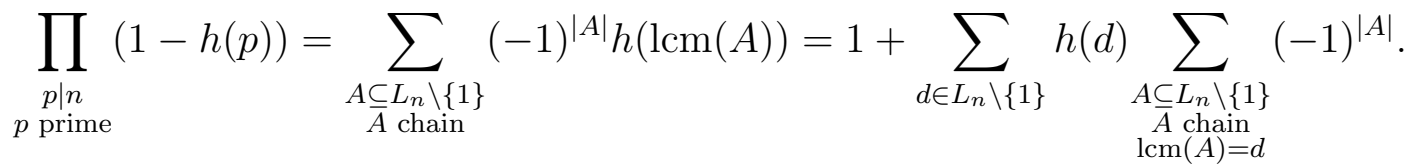

By induction on the height of $d$ in $L_{n} \backslash\{1\}$ it follows that the inner sum in Eq. (38) agrees with $\mu(d)$. Thus, the following known formula (cf. Theorem 2.18 in [1]) is obtained:

$$
\prod_{\substack{p \mid n \\ p \text { prime }}}(1-h(p))=\sum_{d \mid n} h(d) \mu(d) .
$$


Note that in Eqs. (37)- (39) we don't impose any further requirement on $h$. By applying Eq. (39) to $1 / h$ where $h$ is multiplicative and nowhere zero, we rediscover Eq. (36).

\subsubsection{Riemann zeta function}

Closely related to $\varphi_{h}$ in Eq. (33) is the $\zeta$-function, which can be represented as

$$
\frac{1}{\zeta(s)}=\lim _{n \rightarrow \infty} \frac{\varphi_{h}(n !)}{h(n !)}, \quad \operatorname{Re}(s)>1,
$$

where $h(n)=n^{s}$ for any $n \in \mathbb{N}$. By Eq. (33),

$$
\frac{1}{\zeta(s)}=1+\lim _{n \rightarrow \infty} \frac{1}{(n !)^{s}} \sum_{A \subseteq L_{n ! \backslash\{1, n !\}}}(-1)^{|A|}(\operatorname{gcd}(A))^{s}, \quad \operatorname{Re}(s)>1 .
$$

In particular, for $s=2$,

$$
\lim _{n \rightarrow \infty} \frac{1}{(n !)^{2}} \sum_{A \subseteq L_{n ! \backslash\{1, n !\}}}(-1)^{|A|-1}(\operatorname{gcd}(A))^{2}=1-\frac{6}{\pi^{2}} .
$$

Eqs. (40)-(42) also hold if $n$ ! is replaced by $n \#$ where $n \#$ denotes the primorial of $n$, that is, the product of all primes less than or equal to $n$ (sequence A034386 in [19]).

\section{Generalization to convex geometries}

A closure system $(S, h)$ consists of a set $S$ and a hull operator $h$ on $S$, i.e. an extensive, increasing, idempotent operator on subsets of $S$. A subset $A$ of $S$ is called $h$-closed if $h(A)=A$, and $h$-free if all subsets of $A$ are $h$-closed. An $h$-basis of $A$ is a minimal subset $B$ of $A$ such that $h(B)=A$. A convex geometry is a closure system $(S, h)$ where $S$ is finite and any $h$-closed subset of $S$ has a unique $h$-basis [15].

Theorem 2. Let $(S, h)$ be a convex geometry, $\Gamma$ an abelian group (additively written), and $f: 2^{S} \rightarrow \Gamma$ such that for any $h$-closed, but not $h$-free subset $A$ of $S$,

$$
\sum_{I: A_{0} \subseteq I \subseteq A} f(I)=0
$$

where $A_{0}$ denotes the unique h-basis of $A$. Then,

$$
\sum_{A \subseteq S} f(A)=\sum_{\substack{A \subseteq S \\ A \\ h-\text {-free }}} f(A) .
$$

Proof. Since $(S, h)$ is a convex geometry, $h(I)=A$ if and only if $A_{0} \subseteq I \subseteq A$. Hence,

$$
\sum_{A \subseteq S} f(A)=\sum_{\substack{A \subseteq S \\ h \\ h \text {-closed }}} \sum_{I: h(I)=A} f(I)=\sum_{\substack{A \subseteq S \\ A \text {-closed }}} \sum_{I: A_{0} \subseteq I \subseteq A} f(I)=\sum_{\substack{A \subseteq S \\ A \\ h \text {-free }}} \sum_{I: A_{0} \subseteq I \subseteq A} f(I) .
$$

Since any $A \subseteq S$ is $h$-free if and only if $A_{0}=A$, the result follows. 
Remark 3. The requirement in Eq. (43) is satisfied if $f(I)=(-1)^{|I|} \gamma(h(I))$ for any $I \subseteq S$ where $\gamma: 2^{S} \rightarrow \Gamma$. In this case, we obtain

$$
\sum_{A \subseteq S}(-1)^{|A|} \gamma(h(A))=\sum_{\substack{A \subseteq S \\ A \\ h \text {-free }}}(-1)^{|A|} \gamma(A) .
$$

In particular, by defining $\gamma(A)=(-1)^{|A|}$ for any $A \subseteq S$, the sum $\sum_{A \subseteq S}(-1)^{|h(A)|-|A|}$ on the left-hand side of Eq. (45) evaluates to the number of $h$-free subsets of $S$, while by defining $\gamma(A)=1$ for any $A \subseteq S$, Eq. (45) reveals that the Euler characteristic of the abstract simplicial complex of all non-empty $h$-free subsets of $S$ is equal to 1, provided $S \neq \emptyset$. This latter result is attributed to Lawrence (unpublished, cf. [15]).

Remark 4 . The preceding theorem can be generalized even further by requiring that $(S, h)$ is a closure system and replacing Eq. (43) by

$$
\sum_{\emptyset \neq \mathscr{J} \subseteq \mathscr{A}_{0}}(-1)^{|\mathscr{J}|-1} \sum_{I: \cup \mathscr{J} \subseteq I \subseteq A} f(I)=0
$$

where $\mathscr{A}_{0}$ denotes the system of all $h$-bases of $A$. Note that in this more general setting, $A$ is $h$-free if and only if $\mathscr{A}_{0}=\{A\}$.

In the following, we derive Theorem 1 from Theorem 2 .

Proof of Theorem 1. The requirements imply that for any $B \in \mathscr{B}$ there is some $c(B) \in$ $S \backslash B$ such that $B \cup\{c(B)\} \in \mathscr{C}$ and $c(B)>b$ for any $b \in B$. For any $A \subseteq S$ define

$$
\begin{aligned}
\left.\mathscr{B}\right|_{A} & :=\{B \in \mathscr{B} \mid B \subseteq A\}, \\
h(A) & :=A \cup\left\{c(B)|B \in \mathscr{B}|_{A}\right\}, \\
h^{*}(A) & :=h(A) \cup h(h(A)) \cup \ldots
\end{aligned}
$$

Then, $h^{*}$ is a hull operator on $S$, and

$$
A_{0}:=A \backslash\left\{c(B)|B \in \mathscr{B}|_{A}\right\}
$$

is the unique $h^{*}$-basis of any $h^{*}$-closed subset $A$ of $S$.

In order to verify Eq. (43), let $A \subseteq S$ be $h^{*}$-closed, but not $h^{*}$-free. Then, $A_{0} \neq A$ and hence, $\left.\mathscr{B}\right|_{A} \neq \emptyset$. Choose $\left.B^{\prime} \in \mathscr{B}\right|_{A}$ such that $c\left(B^{\prime}\right)=\min \left\{c(B)|B \in \mathscr{B}|_{A}\right\}$. Since $B^{\prime} \subseteq A$ and $A$ is $h^{*}$-closed, $c\left(B^{\prime}\right) \in A$ and therefore, $B^{\prime} \cup\left\{c\left(B^{\prime}\right)\right\} \subseteq A$. We observe that $B^{\prime} \subseteq A_{0}$, since otherwise $B^{\prime} \cap\left\{c(B)|B \in \mathscr{B}|_{A}\right\} \neq \emptyset$, which implies $c(B) \leqslant \max B^{\prime}<c\left(B^{\prime}\right)$ for some $\left.B \in \mathscr{B}\right|_{A}$, contradicting the minimality of $c\left(B^{\prime}\right)$. Now,

$$
\sum_{I: A_{0} \subseteq I \subseteq A} f(I)=\sum_{\substack{I: A_{0} \subseteq I \subseteq A \\ \max C \in I}} f(I)+\sum_{\substack{I: A_{0} \subseteq I \subseteq A \\ \max C \notin I}} f(I)=\sum_{\substack{I: A_{0} \subseteq I \subseteq A \\ \max C \in I}}(f(I)+f(I \backslash\{\max C\}))
$$

where $C:=B^{\prime} \cup\left\{c\left(B^{\prime}\right)\right\}$. Since any $I$ in the latter sum includes $C$, Eq. (3) (with $I$ in place of $A$ ) reveals $f(I)+f(I \backslash\{\max C\})=0$; hence, the whole sum vanishes as required in Eq. (43). Applying Theorem 2 now gives a sum over all $h^{*}$-free subsets of $S$. Since any $A \subseteq S$ is $h^{*}$-free if and only if $B \nsubseteq A$ for any $B \in \mathscr{B}$, the proof is complete. 


\section{References}

[1] T.M. Apostol. Introduction to Analytic Number Theory. New York: Springer-Verlag, 1976.

[2] J.L. Arocha and B. Llano. "Mean value for the matching and dominating polynomial". In: Discuss. Math. Graph Theory 20 (2000), pp. 57-69.

[3] I. Averbouch, J.A. Makowsky, and P. Tittmann. "A graph polynomial arising from community structure". Extended abstract. In: 35th Intern. Workshop on Graphtheoretic Concepts in Computer Science, WG 2009. (Montpellier, France, June 2426, 2009). Ed. by Ch. Paul and M. Habib. Lecture Notes in Computer Science 5911. Berlin: Springer-Verlag, 2010, pp. 33-43.

[4] C. Berge. Hypergraphs: The Theory of Finite Sets. Amsterdam: North-Holland, 1989.

[5] G.D. Birkhoff. "A determinant formula for the number of ways of coloring a map". In: Ann. Math. 14 (1912), pp. 42-46.

[6] F. Blanchet-Sadri et al. "A graph polynomial approach to primitivity". In: 7th International Conference on Languages and Automata Theory and Applications, LATA 2013. (Bilbao, Spain, Apr. 2-5, 2013). Lecture Notes in Computer Science 7810. Heidelberg: Springer-Verlag, 2013, pp. 153-164.

[7] A. Blass and B.E. Sagan. "Möbius functions of lattices". In: Adv. Math. 127 (1997), pp. $94-123$.

[8] H.H. Crapo. "A higher invariant for matroids". In: J. Combin. Theory 2 (1967), pp. 406-417.

[9] R. Diestel. Graph Theory. 4th ed. New York: Springer-Verlag, 2010.

[10] K. Dohmen. "A Broken-Circuits-Theorem for hypergraphs". In: Arch. Math. 64.2 (1995), pp. 159-162.

[11] K. Dohmen. "An improvement of the inclusion-exclusion principle". In: Arch. Math. 72.4 (1999), pp. 298-303.

[12] K. Dohmen, A. Pönitz, and P. Tittmann. "A new two-variable generalization of the chromatic polynomial". In: Discrete Math. Theor. Comput. Sci. 6.1 (2003), pp. 6990 .

[13] K. Dohmen and P. Tittmann. "Domination Reliability". In: Electron. J. Combin. 19 (2012), \#P15.

[14] F.M. Dong, K.M. Koh, and K.L. Teo. Chromatic Polynomials and Chromaticity of Graphs. Singapore: World Scientific, 2005.

[15] P.H. Edelman and R. Jamison. "The theory of convex geometries". In: Geom. Dedicata 19 (1985), pp. 247-270.

[16] R. Glebov, Y. Person, and W. Weps. "On extremal hypergraphs for hamiltonian cycles". In: Europ. J. Combin. 33 (2012), pp. 544-555. 
[17] G. Graetzer. General Lattice Theory. Second edition. Basel: Birkhäuser, 1998.

[18] A.P. Heron. "Matroid polynomials". In: Combinatorics. Ed. by D.J.A Welsh and D.R. Woodall. The Institute of Combinatorics and Its Applications, 1972.

[19] The On-Line Encyclopedia of Integer Sequences. Sequences A00010, A023900, and A034386. URL: http://oeis.org (visited on 10/12/2014).

[20] Y. Liao and Y. Hou. "Note on the subgraph component polynomial". In: Electron. J. Combin. 21 (2014), \#P3.27.

[21] D.Q. Naiman and H.P. Wynn. "Abstract tubes, improved inclusion-exclusion identities and inequalities and importance sampling". In: Ann. Stat. 25 (1997), pp. 19541983.

[22] H. Narushima. "Principle of inclusion-exclusion on semilattices". In: J. Combin. Theory Ser. A 17 (1974), pp. 196-203.

[23] S. Ross. A First Course in Probability. 9th edition. Pearson, 2012.

[24] G.-C. Rota. "On the foundations of combinatorial theory I. Theory of Möbius functions". In: Z. Wahrscheinlichkeitstheorie Verw. Gebiete 2 (1964), pp. 340-368.

[25] R.P. Stanley. "Supersolvable lattices". In: Alg. Univ. 2 (1972), pp. 197-217.

[26] B. Steinbach and C. Posthoff. "Four-Colored Rectangle-free Grids". In: Recent Progress in the Boolean Domain. Ed. by B. Steinbach. Newcastle: Cambridge Scholars Publishing, 2014.

[27] P. Tittmann, I. Averbouch, and J.A. Makowsky. "The enumeration of vertex induced subgraphs with respect to the number of components". In: Europ. J. Combin. 32 (2011), pp. 954-974.

[28] M. Trinks. "A note on a broken-cycle theorem for hypergraphs". In: Discuss. Math. Graph Theory 34 (2014), pp. 641-646.

[29] H. Whitney. "A logical expansion in mathematics". In: Bull. Amer. Math. Soc. 38 (1932), pp. 572-579. 